\title{
A Case of Orbital Emphysema Associated with Frontal Sinus Pneumocele
}

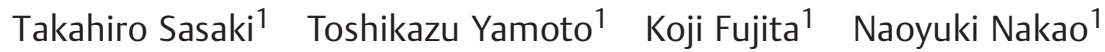 \\ ${ }^{1}$ Department of Neurological Surgery, Wakayama Medical University, \\ Wakayama, Japan \\ Address for correspondence Naoyuki Nakao, MD, PhD, Department of \\ Neurological Surgery, Wakayama Medical University, 811-1 Kimiidera, \\ Wakayama, 641-0012, Japan (e-mail: nnakao@wakayama-med.ac.jp).
}

J Neurol Surg Rep 2013;74:54-56.

\begin{abstract}
Keywords

- orbital emphysema

- frontal sinus pneumocele

- transcranial surgery

Orbital emphysema is usually caused by trauma and fracture of an orbital bone, allowing air to pass from the sinuses into the orbit. Orbital emphysema without any significant trauma is rare. We present a case of a 67-year-old-woman who complained of left exophthalmos without any history of trauma, sneezing, or sinus surgery. Computed tomography scanning showed left orbital emphysema protruding the eyeball forward. The left frontal sinus was remarkably enlarged associated with a partial defect of the orbital roof, allowing air entry into the orbit. In addition, the frontal sinus ostium was occluded with the mucocele that served as a one-way valve between the frontal and the ethmoidal sinuses. We performed frontal craniotomy and removed the mucocele and the inner table of frontal bone to communicate the frontal sinus with the nasal cavity. After operation, her exophthalmos was improved.
\end{abstract}

\section{Introduction}

Orbital emphysema is usually caused by trauma and fracture of an orbital bone, allowing air to pass from the sinuses into the orbit. Orbital emphysema without any significant trauma is rare. We present a case of orbital emphysema with no history of trauma, sneezing, or surgery.

\section{Case Report}

A 67-year-old-woman was admitted to our department complaining of left exophthalmos that had been present and started slowly worsening 1 month ago (-Fig. 1A). There was no history of trauma, sinus disease, sneezing, or sinus surgery. She had no pain, visual disturbance, or double vision. On examination, the visual acuity was $20 / 25$ on the right and $20 / 16$ on the left. The ocular movement was intact. The extent of forward displacement of the eyeball was $15 \mathrm{~mm}$ on the right and $22 \mathrm{~mm}$ on the left by Hertel exophthalmometry.

Computed tomography scanning showed left orbital emphysema, which protruded the eyeball forward ( - Fig. 1B, C). The left frontal sinus was remarkably enlarged associated with a partial defect of the orbital roof. The frontal sinus ostium was

received

February 14, 2013

accepted after revision

March 18, 2013

published online

May 23, 2013 tightly packed with mucus with consequent blockage of the communication with the nasal cavity ( - Fig. 1D).

The patient underwent left frontal craniotomy. The left frontal sinus was enlarged with focal thinning of the bony sinus wall. The orbital roof constituting a part of the frontal sinus wall was thinned and partially lost. The periorbita was observed through the bony defect. After removal of mucus and the frontal sinus mucosa, the sinus ostium was enlarged by drilling the surrounding bone to fully open the blockage of the communication of the frontal sinus with the nasal cavity (-Fig. 2). The partial defect of the orbital roof was not reconstructed. After operation, her exophthalmos was improved. A series of follow-up computed tomography scans for 3 months after surgery showed no appearance of orbital emphysema (- Fig. 3).

\section{Discussion}

Orbital emphysema typically results from traumatic orbital fracture. A rare cause of orbital emphysema is infection, complication of nasal surgery, sneezing, nose blowing, airplane travel, esophageal rupture, and frontal sinus pneumocele. ${ }^{1-4}$
DOI http://dx.doi.org/ 10.1055/s-0033-1347903. ISSN 2193-6358. (c) 2013 Georg Thieme Verlag KG Stuttgart · New York 

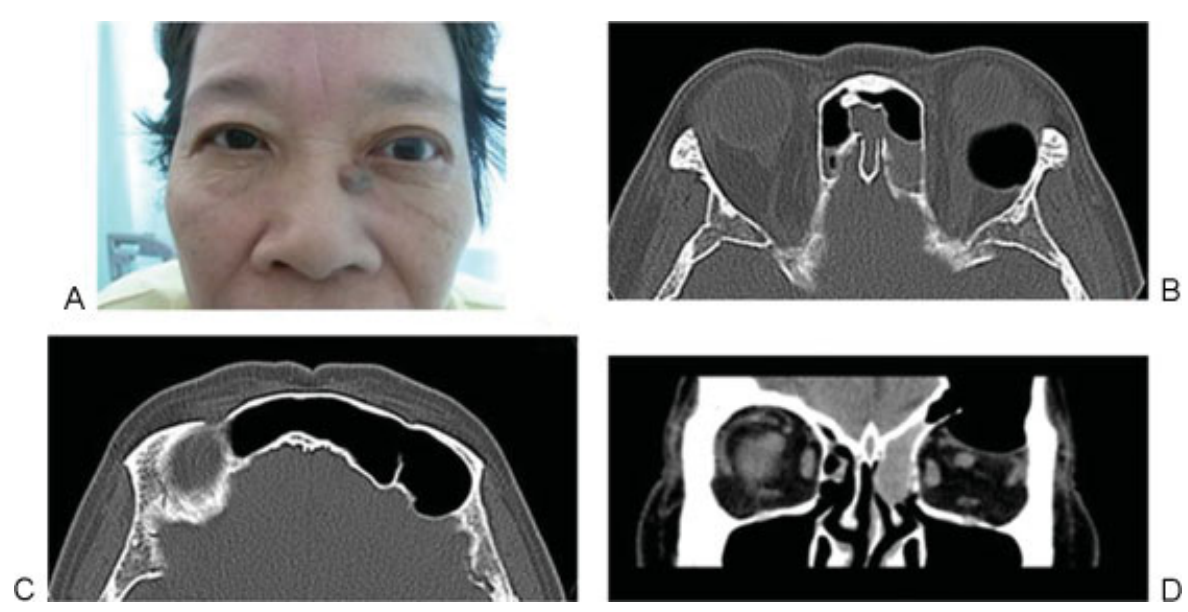

Fig. 1 (A) Left exophthalmos, $15 \mathrm{~mm}$ on the right and $22 \mathrm{~mm}$ on the left by Hertel exophthalmometry. Axial imaging of computed tomography $(B, C)$ showing left frontal sinus pneumocele and orbital emphysema protruding the eye ball forward. Coronal imaging (D) showing that the left frontal sinus was remarkably enlarged and the orbital roof was partially absent. The frontal sinus ostium was occluded with the mucocele that served a one-way valve between the frontal and the ethmoidal sinuses.

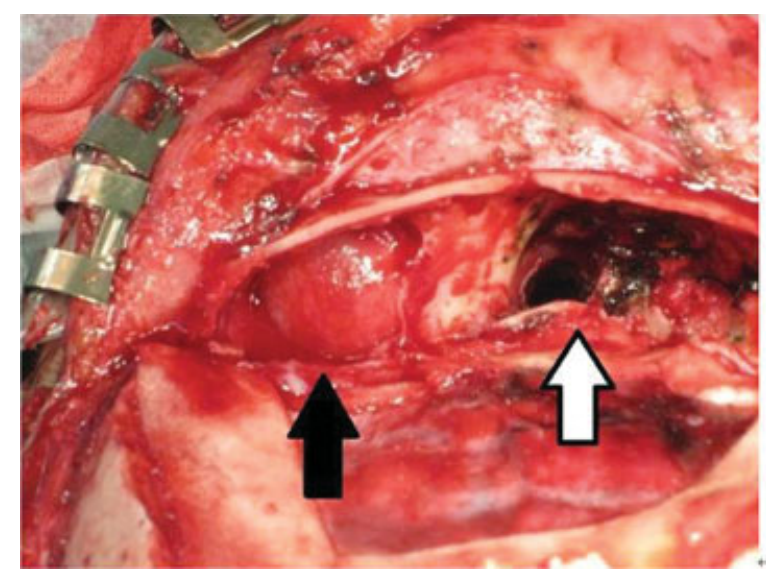

Fig. 2 Surgical view showing that the periorbita was observed through the bony defect (black arrow). After removal of mucus as well as the frontal sinus mucosa, the sinus ostium was enlarged by drilling the surrounding bone to fully open a blockage of the communication of the frontal sinus with the nasal cavity (white arrow).
Frontal sinus pneumocele is characterized by excessive pneumatization of frontal sinus extending beyond the margins of the frontal bone, with thinning and defect of the bony wall and extension of air into the surrounding soft tissue. The accurate pathophysiologic mechanism of development of frontal sinus pneumocele remains to be elucidated. It is proposed that a redundant mucosa or mucocele may serve as a unidirectional valve at the sinus ostium, and that repeated air trapping due to sneezing or nose blowing may result in dilation of the sinus, pressure necrosis. Consequently, this may cause thinning and defect of the sinus wall. ${ }^{3,5}$

In the current case, the patient may originally have had frontal sinus pneumocele that has been proposed a cause of orbital emphysema. ${ }^{3}$ Under such conditions, one can imagine that the patient have would have minor head trauma and light sneezing, triggering this orbital emphysema, even if the patient complained of no such experience.

The management of orbital emphysema associated with frontal sinus pneumocele depends on the clinical presentation and imaging. If there is clinical manifestation of orbital
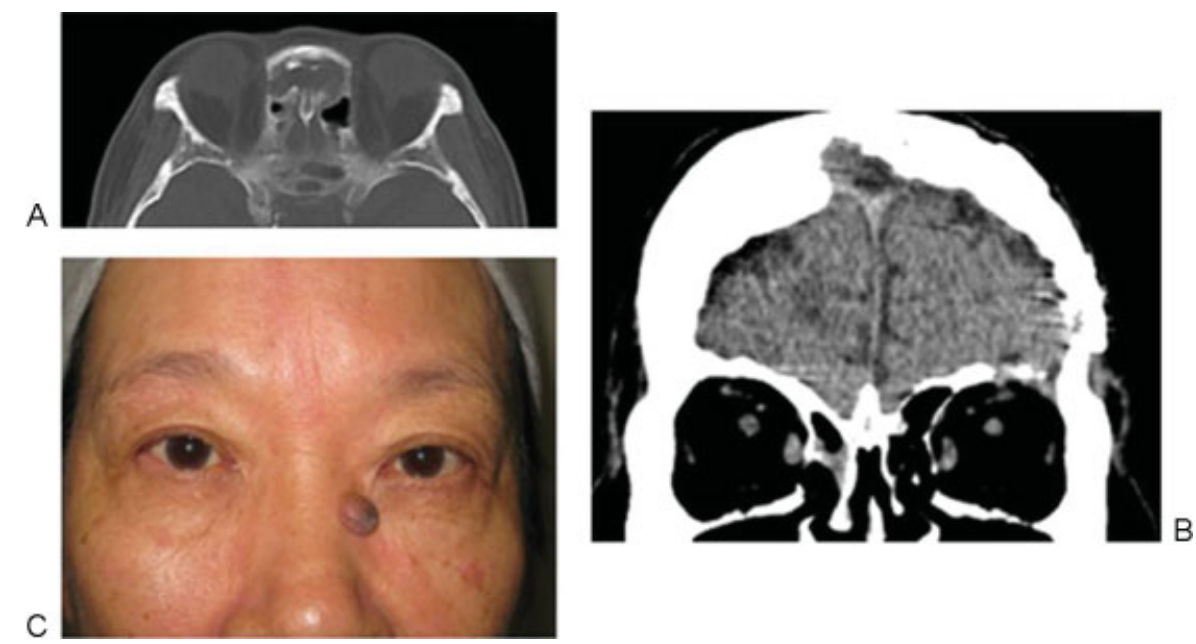

Fig. 3 (A, B) Computed tomography scans and (C) picture after operation showing removal of orbital emphysema and improvement of left exophthalmos. 
compartment syndrome such as sudden orbital pain, visual disturbance, or restriction of ocular motion, emergent decomposition is necessary and can be made by canthotomy or needle aspiration of trapped air. ${ }^{3}$ Other surgical managements have previously been reported, such as frontal sinusotomy with anterior orbitotomy through a subbrow incision ${ }^{3}$ and an endoscopic approach. ${ }^{6}$ On the other hand, Muthiah et al reported that orbital emphysema associated with frontal sinus pneumocele resolved spontaneously as the air was absorbed. ${ }^{7}$ Therefore, conservative management should be considered for nonemergent cases at first.

In our case, we chose surgical intervention for orbital emphysema because the patient complained of exophthalmos on the affected side. We selected a transcranial approach because frontal craniotomy can facilitate drilling of the bone surrounding the sinus ostium and radical removal of mucosa in sinuses. Because an endoscopic sinusotomy can merely remove mucus and thickened mucosa, there is a potential risk of recurrence.

\section{Conclusion}

We present a case of orbital emphysema associated with frontal sinus pneumocele that was treated by a transcranial approach. With a transcranial approach, the sinus ostium can be fully enlarged by drilling the surrounding bone so as to prevent a recurrence of frontal sinus pneumocele.

\section{References}

1 Zimmer-Galler IE, Bartley GB. Orbital emphysema: case reports and review of the literature. Mayo Clin Proc 1994;69(2):115-121

2 Mensiz E, Tüz M, Oyar O, Doğru H, Yasan H. A case of orbital emphysema associated with internal laryngocele. Auris Nasus Larynx 2003;30(2):197-200

3 Boulos PR, Bernardino CR, Rubin PAD. Pneumocele-a rare cause of air in the orbit. Am J Ophthalmol 2004;138(1):168-169

4 Gauguet J-M, Lindquist PA, Shaffer K. Orbital emphysema following ocular trauma and sneezing. Radiology Case Reports. 2008; 3:124-129

5 Benedikt RA, Brown DC, Roth MK, Geyer CA, Ghaed VN. Spontaneous drainage of an ethmoidal mucocele: a possible cause of pneumosinus dilatans. AJNR Am J Neuroradiol 1991;12(4): 729-731

6 Abdel-Aal AK, Abayazeed AH, Raghuram K, Curé JK. Pneumocele of the frontal sinus producing orbital roof defect: case report and review of literature. Am J Otolaryngol 2010;31(3):202-204

7 Muthiah MN, Day AC, Singh RP, Malik N, Adams ME, Olver JM. Frontal sinus pneumocoele: a rare cause of orbital emphysema. Clin Experiment Ophthalmol 2007;35(8):772-773 